\title{
Influência da correção cirúrgica da catarata na percepção laborativa
}

\author{
Influence of cataract surgical correction on working perception
}

Newton Kara-Junior ${ }^{1}$, Marcony Rodrigues Santhiago ${ }^{2}$, Tais Renata Ribeiro Parede ${ }^{1}$, Rodrigo França Espindola ${ }^{1}$, Maysa Godoy Gomes Mazurek ${ }^{1}$, Renato Germano ${ }^{3}$, Newton Kara-Jose ${ }^{1}$

\section{RESUMO}

Objetivo: Analisar o benefício gerado nas atividades profissionais após a cirurgia de catarata.

Métodos: Foi realizado um estudo prospectivo, randomizado, no Hospital das Clínicas da Universidade de São Paulo. Os pacientes foram submetidos à facoemulsificação (FACO) e à extração extracapsular (EECP).

Resultados: A amostra foi composta de 205 pacientes, destes, 101 realizaram cirurgia pela técnica de facoemulsificação. A média de idade no grupo da facoemulsificação foi de 68,3 anos \pm 9 anos e de 69,1 anos $\pm 8,5$ anos no grupo da extração extracapsular $(p=0,70)$. A porcentagem de pacientes empregados no grupo facoemulsificação foi de $16,83 \%$, e no outro grupo de $13,46 \%$. A maioria dos pacientes que não remunerados formalmente sentiu-se motivada a procurar trabalho. A maioria dos analisados relatou aumento da produtividade no trabalho após a cirurgia, 82,50\% no grupo facoemulsificação e 78,60\% no grupo extração extracapsular $(p=0,20)$.

Conclusão: A cirurgia de catarata por ambas as técnicas proporcionou melhora da produtividade no trabalho, e estimulou indivíduos economicamente inativos a procurar trabalho remunerado.

Descritores: Extração de catarata; Facoemulsificação; Catarata/complicações; Cegueira/prevenção \& controle; Conduta na prática dos médicos; Lentes intraoculares; Acuidade visual; Percepção

\begin{abstract}
Purpose: To analyze the benefits after cataract surgery in professional activities.

Methods: A prospective, randomized study was conducted at the Clinical Hospital of the University of São Paulo. The patients had cataract surgery by phacoemulsification (PHACO) and by extracapsular extraction (EECP).

Results: The sample consisted of 205 patients, 101 of these, submitted to phacoemulsification. The mean age in phacoemulsification group was 68.3 years \pm 9 years and 69.1 years \pm 8.5 years in extracapsular extraction group $(p=0.70)$. The percentage of patients employed in phacoemulsification group was $16.83 \%$, and in the extracapsular extraction group of $13.46 \%$. Most patients who were not employed felt motivated to seek work. Most of the patients increased their productivity at work after surgery, $82.50 \%$ in the phacoemulsification group, and $78.60 \%$ in the other group $(p=0.20)$.

Conclusion: Cataractsurgery of both techniques has improved the productivity atworkandencouraged economicallyinactive individuals to seekemployment.
\end{abstract}

Keywords: Cataract extraction; Phacoemulsification; Cataract/complications; Blindness/prevention \& control; Physician's practice patterns; Lenses, intraocular; Vision acuity; Perception

\section{INTRODUÇÃO}

A tualmente a cirurgia de catarata com implante de lente intraocular é um dos procedimentos cirúrgicos mais realizados no mundo(1-3). As técnicas mais utilizadas para a cirurgia de catarata são a extração extracapsular manual do cristalino (EECP) e a facoemulsificação (FACO). Avanços no tratamento têm exigido recursos cada vez maiores. Assim sendo, decisões em saúde pública devem ser baseadas na avaliação da eficiência de cada novo procedimento. Nos últimos anos, em todos os países desenvolvidos, tem ocorrido rápida mudança na técnica utilizada para a cirurgia de catarata, com aumento progressivo do número de cirurgia por facoemulsificação(4).

Trabalho realizado no Setor de Catarata do Departamento de Oftalmologia da Faculdade de Medicina da Universidade de São Paulo - USP.

${ }^{1}$ Médico, Departamento de Oftalmologia, Hospital das Clínicas, Faculdade de Medicina Universidade de São Paulo - USP - São Paulo (SP), Brasil.

2 Médico, Setor de Cirurgia Refrativa, Cleveland Clinic, Cleveland, OH - EUA.

3 Estudante de medicina, Faculdade de Medicina, Universidade de São Paulo - USP - São 3 Estudante de medicin
Paulo (SP), Brasil.

Endereço para correspondência: Marcony Rodrigues Santhiago. $170013^{\text {th }}$ st -44114 , Cleveland, OH, USA - E-mail: marconysanthiago@hotmail.com

Recebido para publicação em 29.05.2009

Última versão recebida em 31.10 .2010

Aprovação em 02.11.2010
A utilização dessa técnica no Sistema Público de Saúde de países em desenvolvimento, apesar de evidências na literatura quanto a seus benefícios clínicos, tem sido desestimulada pela comunidade internacional, devido aos altos custos ${ }^{(5-7)}$. Embora o Sistema Único de Saúde brasileiro (SUS) financie a cirurgia de catarata por FACO, existem questionamentos em relação às vantagens e desvantagens socioeconômicas na realização desse procedimento(8-10).

Diante da escassez de informações na literatura, em relação aos aspectos socioeconômicos da cirurgia de catarata no Sistema Público de Saúde de países em desenvolvimento, foi realizado um estudo com objetivo de avaliar os benefícios gerados com a facectomia nas atividades profissionais.

\section{MÉTODOS}

Foi realizado estudo clínico analítico, prospectivo, randomizado, no Hospital das Clínicas da Faculdade de Medicina da Universidade de São Paulo (HC-FMUSP), em pacientes atendidos em campanhas comunitárias (Projeto Catarata).

Para compor a amostra, foram seguidos os seguintes critérios de inclusão: pessoas de ambos os sexos, entre 41 e 80 anos, portadores de catarata senil, com baixa acuidade visual (AV) bilateral (AV corrigida inferior a 20/40 - Snellen ou 0,3 logMAR, no olho de melhor visão); distância da moradia menor do que 100 km do HC-FMUSP; pressão intraocular inferior a $21 \mathrm{mmHg}$ (sem tratamento) na avaliação inicial; e capacidade de com- 
preender e responder ao questionário a ser aplicado. O questionário, que foi validado previamente, foi aplicado por investigador mascarado quanto ao objetivo do trabalho.

Os critérios de exclusão utilizados foram: restrições físicas ou clínicas, além do problema visual, capazes de limitar o desempenho do indivíduo para as atividades da vida diária e profissional; alterações oculares, além da catarata, que contribuíssem para a diminuição da acuidade visual; cirurgias oculares prévias; história clínica sugestiva de ambliopia; recusa em participar do estudo e assinatura do Termo de Consentimento; e não comparecimento à cirurgia ou aos retornos agendados.

Os pacientes foram divididos, por sorteio, em dois grupos: Grupo 1 ( $n=101)$ : pacientes submetidos à cirurgia de catarata pela técnica de FACO com implante de lente intraocular (LIO) dobrável acrílica Acrysof MA30AC (Alcon Laboratórios); e Grupo $2(n=104)$ : pacientes submetidos à cirurgia de catarata pela técnica de EECP, com implante de LIO rígida de polimetilmetacrilato (PMMA), modelo Cristal Tipo 02 (Alcon Laboratórios). Cada paciente recebeu um número de acordo com a sequência de seleção. A seguir, utilizando-se sorteio aleatório, foi realizada a alocação de cada número a um determinado grupo, a fim de evitar qualquer direcionamento de paciente para algum grupo específico. Os médicos envolvidos no estudo não participaram do sorteio, e tiveram conhecimento da modalidade cirúrgica a ser realizados momentos antes da cirurgia.

Todas as cirurgias foram feitas por três cirurgiões adequadamente treinados, utilizando-se a tecnologia disponível no HC-FMUSP naquele momento. Apenas um olho de cada paciente foi operado.

Todos os testes estatísticos foram realizados com auxílio do programa de computador SPSS (Statistical Package for Social Sciences) 10.0 para Windows TM (SPSS Inc, Chicago, IL, EUA). Os testes Chi-Quadrado e Mann-Whitney foram utilizados para a análise. $O$ valor de $p<0,05$ foi considerado estatisticamente significativo em todos os testes.

\section{RESULTADOS}

Foram submetidos a cirurgia de catarata 205 pacientes, destes, 101 pela técnica de FACO e 104 pela técnica de EECP. A média de idade no grupo da FACO foi de 68,3 anos \pm 9 anos e de 69,1 anos $\pm 8,5$ anos no grupo da EECP $(p=0,70)$. Quanto ao sexo, $35,3 \%$ e $44,1 \%$ dos pacientes eram do sexo masculino no grupo da FACO e EECP, respectivamente $(p=0,40)$. A tabela 1 apresenta a distribuição ocupacional, por tipo de cirurgia. A porcentagem de pacientes empregados no grupo FACO foi de $16,83 \%$, e no grupo EECP de $13,46 \%$

A maioria dos sujeitos não empregados, em ambos os grupos, referiu que a dificuldade visual associada à catarata interferiu na sua situação ocupacional (Tabela 2). Na ocasião da

Tabela 1. Situação ocupacional de pacientes nos grupos de facoemulsificação (FACO) e extração extracapsular (EECP)

\begin{tabular}{|c|c|c|c|c|c|c|}
\hline \multirow{2}{*}{$\begin{array}{l}\text { Situação } \\
\text { ocupacional }\end{array}$} & \multicolumn{2}{|c|}{ FACO } & \multicolumn{2}{|c|}{ EECP } & \multicolumn{2}{|c|}{ Total } \\
\hline & $n$ & $\%$ & $\bar{n}$ & $\%$ & $n$ & $\%$ \\
\hline Aposentado & 49 & 48,04 & 53 & 51,96 & 102 & 100,00 \\
\hline Do lar & 25 & 43,86 & 32 & 56,14 & 57 & 100,00 \\
\hline Empregado & 17 & 54,84 & 14 & 45,16 & 31 & 100,00 \\
\hline$P E^{*}$ & 5 & 55,56 & 4 & 44,44 & 9 & 100,00 \\
\hline NE/NPE** & 5 & 83,34 & 1 & 16,66 & 6 & 100,00 \\
\hline Empregados/total & & 16,83 & & 13,46 & & \\
\hline
\end{tabular}

*= procurando emprego; ${ }^{* *}=$ não empregado/nem procurando emprego alta pós-operatória, a maioria dos pacientes que não tinha remuneração formal, sentiu-se motivada a procurar trabalho (Tabela 3). A maioria dos pacientes relatou aumento da produtividade no trabalho após 180 dias da cirurgia (Tabela 4). Não houve diferença estatisticamente significativa entre os grupos $(p=0,20)$

\section{DISCUSSÃO}

Na presente pesquisa, os dois grupos estudados eram homogêneos, quanto a idade, sexo e tipo de ocupação (Tabela 1). Outros estudos nacionais também observaram características semelhantes de pacientes selecionados para cirurgia de catarata em hospitais públicos ${ }^{(11-12)}$.

Tabela 2. Opinião sobre a influência da dificuldade visual na situação ocupacional dos indivíduos que não trabalhavam remuneradamente, nos grupos de facoemulsificação (FACO) e extração extracapsular (EECP)

\begin{tabular}{|c|c|c|c|c|c|c|c|}
\hline \multirow{3}{*}{$\begin{array}{l}\text { Situação } \\
\text { ocupacional }\end{array}$} & & \multicolumn{6}{|c|}{ Opinião } \\
\hline & & \multicolumn{2}{|c|}{ Sim } & \multicolumn{2}{|c|}{ Não } & \multicolumn{2}{|c|}{ Total } \\
\hline & & $n$ & $\%$ & $n$ & $\%$ & $n$ & $\%$ \\
\hline \multirow[t]{2}{*}{ Aposentado } & FACO & 44 & 89,80 & 5 & 10,20 & 49 & 100,00 \\
\hline & EECP & 43 & 81,13 & 10 & 8,87 & 53 & 100,00 \\
\hline \multirow[t]{2}{*}{ Do lar } & FACO & 21 & 84,00 & 4 & 16,00 & 25 & 100,00 \\
\hline & EECP & 28 & 87,50 & 4 & 12,50 & 32 & 100,00 \\
\hline \multirow[t]{2}{*}{ NE/NPE** } & FACO & 3 & 60,00 & 2 & 40,00 & 5 & 100,00 \\
\hline & EECP & 1 & 100,00 & - & - & 1 & 100,00 \\
\hline \multirow[t]{2}{*}{$P E^{*}$} & FACO & 5 & 100,00 & - & - & 5 & 100,00 \\
\hline & EECP & 4 & 100,00 & - & - & 4 & 100,00 \\
\hline
\end{tabular}

${ }^{*}=$ procurando emprego; ${ }^{* *}=$ não empregado/nem procurando emprego

Tabela 3. Opinião sobre a melhora da dificuldade visual como motivador na procura por emprego de acordo com a situação ocupacional nos grupos analisados

\begin{tabular}{|c|c|c|c|c|c|c|c|c|c|}
\hline \multirow{3}{*}{$\begin{array}{l}\text { Situação } \\
\text { ocupacional }\end{array}$} & & \multicolumn{8}{|c|}{ Opinião } \\
\hline & & \multicolumn{2}{|c|}{$\underset{\text { Sim }}{\text { motivou }}$} & \multicolumn{2}{|c|}{$\begin{array}{c}\text { Não } \\
\text { motivou }\end{array}$} & \multicolumn{2}{|c|}{$\begin{array}{c}\text { Sem } \\
\text { interesse }\end{array}$} & \multicolumn{2}{|c|}{ Total } \\
\hline & & $\bar{n}$ & $\%$ & $\mathbf{n}$ & $\%$ & $\bar{n}$ & $\%$ & $\bar{n}$ & $\%$ \\
\hline \multirow[t]{2}{*}{ Aposentado } & FACO & 44 & 89,80 & - & - & 5 & 10,20 & 49 & 100,00 \\
\hline & EECP & 47 & 88,68 & 1 & 1,89 & 5 & 9,43 & 53 & 100,00 \\
\hline \multirow[t]{2}{*}{ Dolar } & FACO & 22 & 88,00 & 2 & 8,00 & 1 & 4,00 & 25 & 100,00 \\
\hline & EECP & 31 & 96,88 & - & - & 1 & 3,12 & 32 & 100,00 \\
\hline \multirow[t]{2}{*}{ PE/NPE** } & FACO & 5 & 100,00 & - & - & - & - & 5 & 100,00 \\
\hline & EECP & 1 & 100,00 & - & - & - & - & 1 & 100,00 \\
\hline \multirow[t]{2}{*}{$P E^{*}$} & FACO & 4 & 80,00 & - & - & 1 & 20,00 & 5 & 100,00 \\
\hline & EECP & 3 & 75,00 & - & - & 1 & 25,00 & 4 & 100,00 \\
\hline
\end{tabular}

${ }^{*}=$ procurando emprego; ${ }^{* *}=$ não empregado/nem procurando emprego

Tabela 4. Opinião sobre a influência da cirurgia na produtividade dos pacientes empregados nos grupos de facoemulsificação (FACO) e extração extracapsular (EECP)

\begin{tabular}{lccccc}
\hline \multirow{2}{*}{$\begin{array}{l}\text { Produtividade } \\
\text { do trabalho }\end{array}$} & \multicolumn{2}{c}{ FACO } & & \multicolumn{2}{c}{ EECP } \\
\cline { 2 - 3 } \cline { 5 - 6 } & $\mathbf{n}$ & \% & & n & $\%$ \\
\hline Aumentou & 14 & 82,50 & & 11 & 78,60 \\
Diminuiu & - & - & & & - \\
Mesma & 3 & 17,50 & & 3 & 21,40 \\
Total & 17 & 100,00 & & 14 & 100,00 \\
\hline
\end{tabular}


A maior proporção dos indivíduos analisados não exercia atividade profissional remunerada, $83,17 \%$ no grupo FACO e $86,54 \%$ no grupo EECP (Tabela 1), proporção semelhante à constatada por outros estudos ${ }^{(13-14)}$. Estes pacientes relatavam que a atual situação de desemprego era influenciada pela dificuldade visual (Tabela 2). Após a realização da cirurgia, a maioria dos indivíduos não empregados, em ambos os grupos, declarou-se motivada a procurar trabalho, devido à melhora da condição visual (Tabela 3), resultado semelhante a outro estudo nacional(12).

Segundo dados do IBGE em 2002, 36,0\% da população brasileira acima de 50 anos eram economicamente ativa ${ }^{(12)}$ Embora a média de idade dos indivíduos da amostra deste estudo fosse superior a 60 anos, é possível que a proporção dos sujeitos economicamente ativos (média de 15,21\%, em ambos os grupos), seja inferior ao padrão nacional para aquela faixa etária.

Esses indivíduos, apesar da melhora da condição visual após a cirurgia, poderão encontrar dificuldade em conseguir emprego, pelo fato de já terem sido excluídos do mercado de trabalho. Segundo alguns trabalhos ${ }^{(12,14-15)}$, a cirurgia de catarata deve ser realizada antes que o paciente esteja visualmente incapacitado, pois a reintegração ao mercado de trabalho passa a ser um grande obstáculo. A cirurgia realizada antes que as dificuldades resultantes da baixa visão afastem o indivíduo do trabalho significa, também, uma ação preventiva da "cegueira econômica".

Convém enfatizar que em ambos os grupos, a maioria dos indivíduos que estavam trabalhando referiram melhora na produtividade após a cirurgia (Tabela 4). A recuperação visual após a cirurgia de catarata aumenta a produtividade do trabaIhador e estimulam outros que não trabalhavam a procurar trabalho. Esse fato, já pode indicar o retorno ao Estado do investimento com a cirurgia, além de propiciar melhora na qualidade de vida dos pacientes.

Assim, considerando a proporção reduzida de indivíduos que trabalhavam mediante remuneração, pode-se inferir que muitos sujeitos que poderiam ser economicamente ativos, já se incluíam na denominada "cegueira econômica", ocasionando prejuízos para o país, sociedade, para o próprio indivíduo e sua família.

Importante salientar que a amostra estudada foi composta por pacientes selecionados em projetos comunitários (Projeto-Catarata) realizados pelo HC-FMUSP, que visam minimizar as dificuldades de acesso ao tratamento de pacientes com dificuldade visual por catarata. Assim, é possível que, na rotina do atendimento público, existam barreiras adicionais para acesso à cirurgia, retardando ainda mais o tratamento.

\section{CONCLUSÃO}

Nas condições desta pesquisa, a cirurgia de catarata por ambas as técnicas, proporcionou melhora do desempenho no trabalho, e estimulou indivíduos economicamente inativos a procurar trabalho remunerado.

\section{REFERÊNCIAS}

1. Javitt JC, Wang F, West SK. Blindness due to cataract: epidemiology and prevention. Annu Rev Public Health. 1996;17:159-77.

2. Ellwein LB, Kupfer C. Strategic issues in preventing cataract blindness in developing countries. Bull World Health Organ. 1995;73(5):681-90.

3. Thylefors B, Négrel AD, Pararajasegaram R, Dadzie KY. Global data on blindness. Bull World Health Organ 1995;73(1):115-21.

4. Schwab L. Eye care delivery in developing nations: paradigms, paradoxes, and progress. Ophthalmic Epidemiol. 1994;1(3):149-54.

5. Sommer A. Organizing to prevent third word blindness. Am J Ophthalmol 1989; 107(5): 544-6. Comment in: Am J Ophthalmol. 1989;108(4):466-7.

6. Yorston Y. Are intraocular lenses the solution to cataract blindness in Africa? Br J Ophthalmol. 1998:82(5):467-71.

7. Taylor HR, Sommer A. Cataract surgery. A global perspective. Arch Ophthalmol. 1990;108(6):797-8.

8. Leinonen J, Laatikainen L. Changes in visual acuity of patients undergoing cataract surgery during the last two decades. Acta Ophthalmol Scand. 2002;80(5):506-11.

9. Lundstrom M, Stenevi U, Thorburn W. The Swedish National Cataract Register: a 9-year review. Acta Ophthalmol Scand. 2002;80(3):248-57.

10. Schein OD, Steinberg EP, Javitt JC, Cassard SD, Tielsch JM, Steinwachs DM, et al. Variation in cataract surgery practice and clinical outcomes. Ophthalmology. 1994;101(6):1142-52.

11. Kara-José N, Barbosa E, Fonseca-Neto JC, Oura MH, Martins WH. Considerações sobre aspectos sociais do atendimento clínico e cirúrgico de pacientes portadores de catarata senil. Arq Bras Oftalmol. 1982;45(4):115-8.

12. Temporini ER, Kara-José N, Kara-Junior N. Catarata senil: características e percepções de pacientes atendidos em projeto comunitário de reabilitação visual. Arq Bras Oftalmol. 1997;60(1):79-83.

13. Kara-José N, Contreras F, Campos MA, Delgado AMN, Mowery RL, Ellwein LB. Screening and surgical intervention results from cataract-free-zone projects in Campinas, Brasil and Chimbote, Peru. Int Ophthalmol. 1990;14(3):155-64.

14. Kara-Junior N, Schellini SA, Silva MRBM, Bruni LF, Almeida AGC. Projeto Catarata Qual a sua importância para a comunidade? Arq Bras Oftalmol. 1996;59(5):490-6.

15. Albanis C, Dwyer MA, Ernest JT. Outcomes of extracapsular cataract extraction and phacoemulsification performed in a University training program. Ophthalmic Surg Lasers. 1998;29(8):643-8.

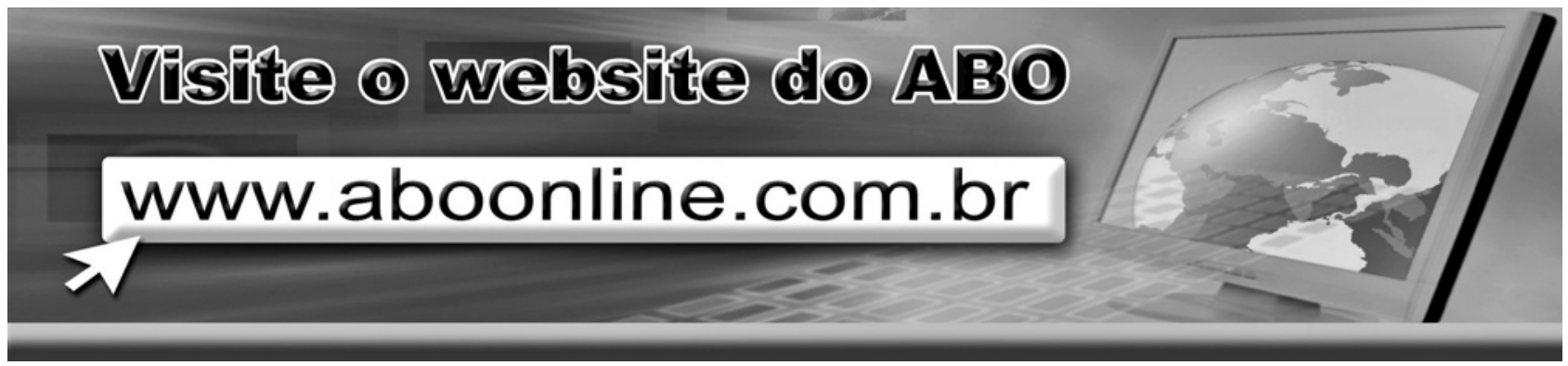

